\title{
Disposable Zero-Knowledge Authentications and Their Applications to Untraceable Electronic Cash
}

\author{
Tatsuaki Okamoto Kazuo Ohta
}

NTT Communications and Information Processing Laboratories

Nippon Telegraph and Telephone Corporation

1-2356, Take, Yokosuka-shi, Kanagawa-ken, 238-03, Japan

\section{Abstract}

In this paper, we propose a new type of authentication system, disposable zero-knowledge authentication system. Informally speaking, in this authentication system, double usage of the same authentication is prevented. Based on these disposable zero-knowledge authentication systems, we propose a new untraceable electronic cash scheme satisfying both untraceability and unreusablity. This scheme overcomes the problems of the previous scheme proposed by Chaum, Fiat and Naor through its greater efficiency and provable security under reasonable cryptog raphic assumptions. We also propose a scheme, transferable untraceable electronic cash scheme, satisfying transferability as well as the above two criteria, whose properties have not been previously proposed in any other scheme. Moreover, we also propose a new type of electronic cash, untraceable electronic coupon ticket, in which the value of one piece of the electronic cash can be subdivided into many pieces.

\section{Introduction}

"Zero-knowledge proofs" are useful for many application areas [B, BC, BCC, $\mathrm{Cr}, \mathrm{GMR}$, etc.]. A typical application area is authentication systems such as identifications and digital signatures [FFS, FS, GQ, MS, OhO]. For zero-knowledge proofs, coin flips of the prover are essential for zero-knowledgeness of the proof, while coin flips of the verifier are essential for the ability of the proof. Therefore, if the coin flips of the prover are restricted, the usage of the proof must be restricted under the zero-knowledge condition.

In this paper, by using this property of zero-knowledge proofs, we propose a new type of zero-knowledge authentication, disposable zero-knowledge authentication. Informally speaking, in this authentication double usage of the same authentication is prevented. This type of zero-knowledge authentication is considered to have many applications such as electronic cash, checks, and tickets, because in these applications a piece of information has value itself, and multiple invalid usage of this piece of information must be prevented.

To endow electronic cash with properties similar to those of real cash, electronic cash should satisfy the following conditions:

(1) Untraceability: The privacy of the user should be protected. That is, the relationship between the user and his purchases should be untraceable by anyone. Ideally, any partial information about the user's purchases should be untraceable by anyone (we call this property perfect untraceability). 
(2) Unreusability: The ability to use the electronic cash more than once should be prevented.

(3) Transferability: The electronic cash can be transfered to other users.

Criterion (1) can be satisfied by using blind signatures [Ch, D, OkO]. To satisfy criteria (1) and (2), Chaum, Fiat and Naor [CFN] have developed an elegant electronic cash scheme (untraceable electronic cash) based on the cut-and-choose methodology and collision free functions. However, their scheme has two major problems from the viewpoint of efficiency and formal provability of security:

- (Efficiency) In their scheme, a customer must undergo a complex procedure including the cut-and-choose methodology to obtain each electronic coin.

- (Provability of security) The assumptions under which the scheme is provably secure are not clear.

Although the scheme [CFN] satisfies the first two criteria, so far no scheme has been proposed that satisfies all three criteria: (1) untraceability, (2) unreusability, and (3) transferability.

In this paper, we propose a new untraceable electronic cash scheme that satisfies the criteria (1) and (2) based on disposable zero-knowledge authentications as well as the cut-and-choose methodology. This scheme overcomes the problems of the previous scheme [CFN] in the following ways:

- (Efficiency) In our scheme, a customer undergoes a complex procedure including the cut-and-choose methodology only once when he opens his account at a bank. After that, only a minimal procedure is required to obtain each electronic coin. In this case, however, our scheme does not satisfy perfect untraceability, although it satisfies untraceability. That is, the relationship between a user and his purchases cannot be traced by anyone, but a purchase history of an anonymous user can be traced.

Note that, in our scheme, we can choose the degree of efficiency and the degree of untraceability, and they have a trade-off. If we choose the degree of untraceability as maximal (or perfect untraceability), the degree of efficiency is minimal (or comparable to that of the previous scheme [CFN]).

- (Provability of security) Our scheme is proven to be secure under the following assumptions:

(i) (Digital signature assumption) There exists a secure digital signature scheme [GoMiRi] and secure multiple blind digital signature scheme. (If a secure multiple blind digital signature scheme exists, a secure digital signature scheme exits.)

(ii) (RSA assumption) The RSA scheme is secure, and to break the RSA scheme in which the plaintext's redundancy is 0 is as hard to break as the RSA scheme in which the plaintext's redundancy is less than $1 / 2$. (If the latter condition holds, the former condition holds.)

In this paper, we show a typical case where we construct our scheme based on the extended Fiat-Shamir scheme [GQ, OhO]. Note, however, that our scheme can be constructed based on other disposable zero-knowledge authentications with a unique solution $S$ for $I$ such that $(I, S) \in R$ (e.g., discrete logarithm problem). 
In this case, the second assumption for the scheme's security is replaced by the following one (The first assumption is the same as above) :

(ii) (Invulnerable relation assumption) A relation $R$ is invulnerable (it is hard to compute $S$ from $I$, where $(I, S) \in R$ ). And, to compute $S$ from $I$ in which $S$ 's redundancy is 0 is as hard to compute as $S$ from $I$ in which $S$ 's redundancy is less than $1 / 2$.

Next, we propose a new electronic cash scheme, transferable untraceable electronic cash scheme that, to our knowledge, has never been proposed, that satisfies criteria (1), (2) and (3). This scheme is constructed based on the above untraceable electronic cash scheme.

Moreover, we also propose another type of electronic cash (untraceable electronic coupon ticket) with the following property: The value of one piece of electronic cash can be subdivided into many pieces. For example, a user with a piece of electronic cash worth $\$ 100$ could subdivide it into 100 pieces of cash worth \$1. If we add the notion of transferability (criterion (3)), transferable untraceable electronic coupon ticket could be constructed in a way similar to the transferable untraceable electronic cash.

\section{Notations}

$(P, V)$ is an interactive pair of Turing machines, where $P$ is the prover, and $V$ is the verifier [GMR, TM]. Let $T \in\{P, V\} . T(s)$ denotes $T$ begun with $s$ on its input work tape. $(P, V)(I)$ refers to the probability space that assigns to the string $\sigma$ the probability that $(P, V)$, on input $I$, outputs $\sigma$.

$(P(s), \underline{V(t)})(I), V$ 's history, denotes $\left(I, t, \rho^{\prime}, m^{\prime}\right)$, where $\rho^{\prime}$ is the finite prefix of $V$ 's random tape that was read, and $m^{\prime}$ is the final content of the communication channel tape on which $P$ writes. $P^{A}$ means $P$ with oracle $A$, where $P^{A}$ 's oracle tapes correspond to $P$ 's communucation channel tapes with $A . R \subseteq X \times Y$ is a relation, where $X$ and $Y$ are sets of finite strings. \| denotes concatenation.

\section{Disposable Zero Knowledge Authentication}

There are two types of interactive proofs. One is the interactive proof for membership in language $L$, in which a membership of an instance in language $L$ is demonstrated [GMR]. The other is the interactive proof for possession of knowledge, in which a prover's possession of information is demonstrated [FFS, $\mathrm{TW}$. In the latter proof, the prover's power is bounded in polynomial time, while, in the former proof, its power is not bounded.

In this section, first, we show the revealability of the zero-knowledge interactive proofs for possession of knowledge. Then, we define the disposable zero-knowledge authentication and show an implementation of this authentication.

Definition 1. Let $R$ be a relation, and $(P, V)$ be a zero-knowledge interactive proof for possession of some $S$ satisfying $(I, S) \in R$. We say that $(P, V)$ is $k$ revealable if there exists a polynomial-time probabilistic Turing machine $M_{V}$ (with complete control over $V)$ that computes $S^{\prime}$ such that $\left(I, S^{\prime}\right) \in R$ after $k$ executions 
of the proof fixing the coin flips $\rho$ of $P$ with overwhelming probability, where $\rho$ corresponds to one execution of the proof. (When $k=O\left(|I|^{c}\right.$ ) and $c$ is a constant, $k$-revealable is called poly-revealable.)

Lemma 1. Every zero-knowledge interactive proof for possession of knowledge is poly-revealable.

Proof Sketch: From the definition of the soundness of the zero-knowledge interactive proof for possession of knowledge [FFS], the existence of $M_{V}$ can be shown. QED

Lemma 2. If there exist secure encryption schemes, any $N P$ relation has a 2-revealable zero-knowledge interactive proof for possession of knowledge.

Proof Sketch: Under the assumption that NP reductions are one-to-one and efficiently invertible, it suffices to prove that Blum's zero-knowledge interactive proof for possession of knowledge of the graph Hamiltonicity ([FFS]'s Theorem 1.) is 2-revealable. The iterated number of rounds of this proof is $|I|$ (one round means steps 1-7 of [FFS]'s Theorem 1.). Suppose that Blum's proof $(P, V)$ is executed twice fuxing the coin flips $\rho$ of $P$. Let $\rho_{i}^{\prime}(i=1,2)$ be the verifier's coin flip in the $i$-th execution of the proof, where $\left|\rho_{i}^{\prime}\right|=|I|$. If $\rho_{1}^{\prime} \neq \rho_{2}^{\prime}$, a Hamiltonian cycle $S$ can be computed in polynomial-time from the history of the two executions of this proof. The probablity that $\rho_{1}^{\prime} \neq \rho_{2}^{\prime}$ is $1-1 / 2^{|I|}$. QED

Definition 2. Let $A$ be the authority, $P_{i}$ be a prover with a secret knowledge $S$ such that $(I, S) \in R$, and $V_{i}$ be a verifier with public knowledge $I$ and $R$. Each party follows the below authentication procedure:

(Step 1) Prover $P_{i}$ sends a message $X$ to authority $A$.

(Step 2) $A$ generates a digital signature $C$ of $X$ and sends it to $P_{i} .(C, X)$ means $A$ 's permission to $P_{i}$ 's authentication. One permission corresponds to one execution of the prover's authentication procedure.

(Step 3) $P_{i}$ shows $A$ 's permission $(C, X)$ and proves his possession of $S$ to a verifier $V_{i} . V_{i}$ accepts $P_{i}$ to be valid if he verifies the validity of both $(C, X)$ and his proof of possession of $S$.

Let $\bar{P}_{i}$ and $\bar{V}_{i}$ be valid prover and verifier that follow their designated protocols, respectively. Let $\widetilde{P}_{i}$ and $\widetilde{V}_{i}$ be invalid polynomial-time prover and verifier that can deviate from their correct protocols in arbitrary ways, respectively. Let $P_{i}$ be either $\bar{P}_{i}$ or $\widetilde{P}_{i}$, and $V_{i}$ be either $\bar{V}_{i}$ or $\widetilde{V}_{i}$.

Let $\left(A,\left\{P_{i}, V_{i}\right\}\right)(i=1,2, \ldots)$ be an authentication system, if the following two conditions are satisfied.

- Completeness: $\bar{P}_{i}(S)$ 's authentication is accepted to be valid by $\bar{V}_{i}$ with overwhelming probability.

- Soundness: There exists a polynomial-time probabilistic Turing machine $M_{P_{i}}$ (with complete control over $P_{i}$ ) such that if $P_{i}$ 's authentication is accepted to be valid by $\bar{V}_{i}$ with non-negligible probability, then $M_{P_{i}}$ breaks cryptographic assumptions on $A$ 's digital signatures with overwhelming probability and the output produced by $M_{P_{i}}$ on input $I$ satisfies the relation $R$ with overwhelming probability. 
Note: Informally, soundness means that invalid prover $\widetilde{P}_{i}$ is accepted by $\bar{V}_{i}$ with negligible probability.

Definition 3. Authentication system $\left(A,\left\{P_{i}, V_{i}\right\}\right)$ is disposable zero-knowledge if the following conditions are satisfied.

- Zero-knowledgeness: For any $V_{i}, I$, and $t$, there exists a polynomial-time probabilistic Turing machine $M_{V_{i}}^{A}$ such that $\left((C, X),\left(\bar{P}_{i}(S), \underline{V_{i}(t)}\right)(I)\right)$ and $M_{V_{i}}^{A}(I, t)$ are polynomially indistinguishable.

- Disposability: There exists a polynomial-time probabilistic Turing machine $M_{\bar{V}_{i}, \bar{V}_{j}}$ (with complete control over $\bar{V}_{i}$ and $\bar{V}_{j}$ ) such that if $\bar{P}_{i}$ 's authentication is executed successfully twice with the same $(C, X)$ to $\bar{V}_{i}$ and $\bar{V}_{j}$ respectively, then the output produced by $M_{\bar{V}_{i}, \bar{V}_{j}}$ on input $I$ satisfies the relation $R$ with overwhelming probability.

Note: Informally, zero-knowledgeness means that when ralid prover $\bar{P}_{i}$ 's authentication is executed once with the same $(C, X)$, then any knowledge about secret information $S$ cannot be revealed by anyone. Disposability means that valid prover $\bar{P}_{i}$ 's authentication is executed twice with the same $(C, X)$, then secret information $S$ can be revealed by the coalition of the authority and valid verifiers.

Theorem 1. If there exist secure encryption schemes [GM] and secure digital signature schemes [GoMiRi], then a disposable zero-knowledge authentication system can be constructed using any $N P$ relation.

Proof Sketch: Under the assumption that NP reductions are one-to-one and efficiently invertible, it suffices to prove that a disposable zero-knowledge authentication system can be constructed using Blum's zero-knowledge interactive proof for graph Hamiltonicity.

Construction:

The public input $I$ is a graph, and $S$ such that $(I, S) \in R$ is a Hamiltonian cycle in $I . H$ is a probabilistic encryption [GM, Y].

(Step 1) $P_{i}$ randomly permutes the vertices of graph $I$ (using permutation $\pi_{k}$, $k=1,2, \ldots,|I|)$ to obtain

- Graph $\hat{I}_{k}$,

- An $|I| \times|I|$ matrix $\alpha_{k}=\left\{\alpha_{k s t}|s, t=1,2, \ldots| I \mid,\right\}$, where $\alpha_{k s t}=H\left(v_{k s t}\right)$, and $v_{k s t}=1$ if edge st is present in the $\widehat{I}_{k}$, and 0 otherwise, and

- $\beta_{k}=H\left(\pi_{k}\right)$.

$P_{i}$ sends $X=\left(\alpha_{1}, \ldots, \alpha_{|I|}, \beta_{1}, \ldots, \beta_{|I|}\right)$ to authority $A$.

(Step 2) $A$ generates a digital signature $\left\{C_{k}\right\}$ of $\left\{\left(\alpha_{k}, \beta_{k}\right)\right\}(k=1,2, \ldots,|I|)$ and sends them to $P_{i}$.

(Step 3-1) $k \leftarrow 1$.

(Step 3-2) $P_{i}$ sends $\left(\alpha_{k}, \beta_{k}\right)$ and $C_{k}$ to $V_{i}$.

(Step 3-3) $V_{i}$ verifies the validity of the digital signature of $C_{k}$. If it is invalid, $V_{i}$ rejects. Otherwise, $V_{i}$ chooses at random $\rho_{k}^{\prime} \in\{0,1\}$, and sends $\rho_{k}^{\prime}$ to $P_{i}$.

(Step 3-4) If $\rho_{k}^{\prime}=1, P_{i}$ sets $\delta_{k}=$ (decryptions of $\alpha_{k s t}$ and $\beta_{k}$ ). Otherwise, $\delta_{k}=\left\{\right.$ decryption of $\alpha_{k s t} \mid$ edge $s t$ is in a Hamiltonian path in $\left.\widehat{I}_{k}\right\} . P_{i}$ sends $\delta_{k}$ to $V_{i}$. 
(Step 3-5) If $P_{i}$ is unable to perform steps 3-4 correctly, $V_{i}$ rejects. Otherwise, $k \leftarrow k+1$ and go to step $3-2$, if $k<|I|$. If $k=|I|, V_{i}$ accepts.

Completeness: A valid prover can be accepted by a verifier with probability 1.

Soundness: If $P_{i}$ 's authentication is accepted to be valid by $\bar{V}_{i}$ with non-negligible probability, then $P_{i}$ can generate $A$ 's digital signature for non-negligible fraction of the message space. Therefore, from the assumption of the existence of secure digital signature, there exists a polynomial-time probabilistic Turing machine $M_{P_{i}}$ (with complete control over $P_{i}$ ) such that $M_{P_{i}}$ breaks cryptographic assumptions of $A$ 's digital signature with overwhelming probability. On the other hand, if $P_{i}$ 's authentication is accepted to be valid by $\bar{V}_{i}$ with non-negligible probability, then, from the soundness of Blum's protocol, $M_{P_{i}}$ on input $I$ outputs $S^{\prime}$ such that $\left(I, S^{\prime}\right) \in R$ with overwhelming probability.

Zero-knowledgeness: In a manner similar to the proof of the zero-knowledgeness

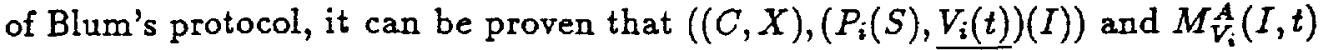
are polynomially indistinguishable.

Disposability: From Lemma 2., we can show that there exists a polynomial-time probabilistic Turing machine $M_{V_{i}}$ that produces $S^{\prime}$ satisfying $\left(I, S^{\prime}\right) \in R$ with overwhelming probability. QED

An application to traceable electronic cash: Here, we show a straightforward application example of the disposable zero-knowledge authentication to traceable electronic cash sytems. Let authority $A$ be a bank, prover $P_{i}$ be a customer, and verifier $V_{i}$ be a shop. The authority's permission $(C, X)$ corresponds to an electronic coin worth $\$ 100$ that the bank issues to the customer when he withdraws $\$ 100$ from his account. When $P_{i}$ purchases an article for $\$ 100$ from shop $V_{i}$, he makes the disposable zero-knowledge authentication regarding $(C, X)$ to the shop. The shop sends the history of this authentication with $(C, X)$ to the bank to obtain $\$ 100$ from the bank. If the customer uses $(C, X)$ more than once, the bank will reveal $S^{\prime}$ satisfying $\left(I, S^{\prime}\right) \in R$, and withdraw more than the $\$ 100$ from his account to penalize the customer. Otherwise, $S^{\prime}$ is never revealed by anyone. Here, $S^{\prime}$ witnesses the customer's abuse of the electronic cash, and we assume that the bank and the customer have signed a contract such that the customer must pay a penalty to the bank when the bank reveals $S^{\prime}$ satisfying $\left(I, S^{\prime}\right) \in R$. In other words, we can consider this situation as a game between the bank and the customer. In this game, the customer loses and pay some money to the bank when the bank reveals $S^{\prime}$ satisfying $\left(I, S^{\prime}\right) \in R$, where $I$ is determined by the customer.

Discussion: The above-mentioned example is an application to a traceable electronic cash system, in which the customer's purchase history is traceable. Although the above-mentioned example is very simple and efficient, we can easily construct other implementations with almost the same functions by using digital signatures of the customer without using disposable zero-knowledge authentication.

In contrast to this application, a number of distinct advantages can be realized by 
applying disposable zero-knowledge authentication to untraceable electronic cash and tickets systems. We will describe these applications in some detail in sections 4,5 and 6.

\section{Untraceable Electronic Cash}

In this section, we show an untraceable electronic cash scheme satisfying two criteria, Untraceability and Unreusability, based on disposable zero-knowledge authentications. This scheme has advantages over the previously proposed scheme [CFN] in the standpoints of efficiency and provability of its security, as described in Section 1. This scheme can be constructed based on some disposable zeroknowledge authentications with a unique solution $S$ for $I$ such that $(I, S) \in R$. In this section, however, we show a typical case based on the extended Fiat-Shamir scheme [GQ, OhO].

Before describing the untraceable electronic cash protocol, we will introduce a specific type of blind digital signature, multiple blind digital signature.

Definition 4. Let $A, P$, and $e_{A}$ be a signer, requester, and the signer's public key, respectively. Let $F$ be an algorithm for $P, D$ be an algorithm for $A$, and $G_{e_{1}}\left(m_{1}, \ldots, m_{k}\right)$ be $A$ 's multiple digital signature of $k$ messages, $m_{1}, \ldots, m_{k}$.

Let $(A, P, F, D, G)$ be a multiple blind digital signature system, if $A$ and $P$ follow the below procedure:

(Step 1) $P$ generates $k$ blind messages $\left\{F_{e_{A}}\left(m_{i}\right) \mid i=1,2, \ldots, k\right\}$ from $k$ messages $\left\{m_{i} \mid i=1,2, \ldots, k\right\}$, and sends them to $A$. Here, each $F_{e_{1}}\left(m_{i}\right)$ is independently blinded.

(Step 2) $A$ generates the multiple blind digital signature $D_{e_{A}}\left(F_{e_{A}}\left(m_{1}\right), \ldots, F_{e_{A}}\right.$ $\left(m_{k}\right)$ ) from the $k$ blind messages, $F_{e_{A}}\left(m_{1}\right), \ldots, F_{e_{A}}\left(m_{k}\right)$, and sends it to $P$.

(Step 3) $P$ extracts $A$ 's multiple digital signature $G_{e_{\Lambda}}\left(m_{1}, \ldots, m_{k}\right)$ of $m_{1}, \ldots, m_{k}$. $(A, P, F, D, G)$ is a secure multiple blind digital signature system, if $(A, P, F, D, G)$ satisfies the criteria for blind digital signature $[\mathrm{Ch}, O k O]$ and $P$ can generate $G_{e_{\Lambda}}\left(m_{1}, \ldots, m_{k}\right)$ from $D_{e_{\Lambda}}\left(m_{1}^{(1)}, \ldots, m_{t}^{(1)}\right), \ldots, D_{e_{\Lambda}}\left(m_{1}^{(l)}, \ldots, m_{t}^{(l)}\right)$ with negligible probability, where $k, t, l$ are positive integers, and for any subset $\left\{i_{1}, \ldots, i_{j}\right\} \subset$ $\{1, \ldots, l\},\left\{m_{1}, \ldots, m_{k}\right\} \neq\left\{m_{1}^{\left(i_{1}\right)}, \ldots, m_{t}^{\left(i_{1}\right)}, \ldots, m_{1}^{\left(i_{i}\right)}, \ldots, m_{t}^{\left(i_{i}\right)}\right\}$, and every $m_{i}$ 's and $m_{i}^{(j)}$ 's are in a randomly determined negligible fraction $\mathcal{M}$ of the message space (e.g., $\mathcal{M}=\{m|| m \mid / c$-prefix of $m$ is a (randomly) fixed sequence, where $c$ is a constant \}).

Note: The secure multiple blind digital signature schemes seem to be implemented based on the previously proposed blind digital signature schemes [ $\mathrm{Ch}$, OkO]. Note that the multiple blind digital signature scheme contains the previous (single) blind digital signature scheme as a special case where $k=1$. In the electronic cash scheme [CNF], the multiple blind digital signature scheme based on $[\mathrm{Ch}]$ has been used. We can also construct a multiple blind digital signature scheme based on a divertible zero-knowledge proof for endomorphic CRSR relation [OkO]. Here, note that, in this scheme based on [OkO], $A$ must send a pre-message to $P$. However, for simplicity in this paper, we omit this pre-message sending phase 
when describing the multiple blind digital signature.

Protocol 1. (Untraceable electronic cash) Bank $A$ has a secure multiple blind digital signature generation algorithm $D$. $A$ has published his public keys, $e_{A}, e_{A}^{\prime}$, of this blind digital signature scheme, where $e_{A}$ corresponds to the electronic license that $A$ issues, and $e_{A}^{\prime}$ corresponds to the value of the electronic coin that $A$ issues. The bank $A$ also sets the security parameter $K=O(|n|)$. Customer $P$ has a bank account number $I D_{P}$ and a secure digital signature generation algorithm $G$, and publishes his public-key $e_{P}$ of the digital signature scheme.

\section{Part I.}

When a customer $P$ opens an account at bank $A, A$ issues an electronic license $B$ to use electronic cash of bank $A$. (Precisely, an electronic license is $\left(B,\left\{I_{i}, N_{i}, L_{i}\right\}\right)$. For simplicity, however, we call $B$ an electronic license.) To get $B, P$ conducts the following protocol with $A$. This procedure is executed only once when $P$ opens the account, unless $P$ reuses the electronic cash invalidly.

(Step 1) Customer $P$ chooses random values $a_{i}$, and composite numbers $N_{i}$ with two large prime factors $P_{i}, Q_{i}\left(N_{i}=P_{i} \cdot Q_{i}\right)$, for $i=1, \ldots, K . P$ also fixes prime integer $L_{i}$ such that $\operatorname{gcd}\left(L_{i}, \phi\left(N_{i}\right)\right)=1$, where $\phi\left(N_{i}\right)=\operatorname{lcm}\left(P_{i}-1, Q_{i}-1\right)$. For simplicity, we assume that all $\left|N_{i}\right|=O\left(\left|e_{A}\right|\right)(i=1, \ldots, K)$ are equivalent, and that $L_{i}=O(1)$.

(Step 2) $P$ forms and sends $K$ blind candidates $W_{i}(i=1, \ldots, K)$ to bank $A$.

$$
W_{i}=F_{e_{1}}\left(I_{i}\left\|N_{i}\right\| L_{i}\right) \quad \text { for } 1 \leq i \leq K
$$

where

$$
\begin{gathered}
I_{i}=S_{i}^{L_{i}} \bmod N_{i}, \\
S_{i}=I D_{P}\left\|a_{i}\right\| G_{e_{\mathrm{P}}}\left(I D_{P} \| a_{i}\right) .
\end{gathered}
$$

(Step 3) $A$ chooses a random subset of $K / 2$ blind candidates indices $U=\left\{i_{j}\right\}, 1 \leq$ $i_{j} \leq K$ for $1 \leq j \leq K / 2$ and transmit it to $P$.

(Step 4) $P$ displays the $a_{i}, P_{i}, Q_{i}, L_{i}, G_{e_{P}}\left(I D_{P} \| a_{i}\right), I D_{P}$ for all $i$ in $U$, and random values that make messages $W_{i}$ blinded, then $A$ checks them. If they are not valid, $A$ halts this protocol.

To simplify notations, we will assume that $U=\{K / 2+1, K / 2+2, \ldots, K\}$. (Step 5) $A$ gives $P$

$$
D_{e_{\Lambda}}\left(W_{1}, \ldots, W_{K / 2}\right) \text {. }
$$

(Step 6) $P$ can then extract the electronic license $B$.

$$
B=G_{e_{A}}\left(I_{1}\left\|N_{1}\right\| L_{1}, \quad \cdots \quad, I_{K / 2}\left\|N_{K / 2}\right\| L_{K / 2}\right) .
$$

Notes:

(1) Every $L_{i}$ 's for every customers can be replaced by a unique prime integer $L$ determined by the system (or a bank). Note that in this case $P$ must select $N_{i}$ such that $\operatorname{gcd}\left(L, \phi\left(N_{i}\right)\right)=1$. 
(2) For example, when we use the RSA multiple blind digital signature scheme [Ch, CFN], $B=\prod_{1 \leq i \leq K / 2} g\left(I_{i}\left\|N_{i}\right\| L_{i}\right)^{1 / e} \bmod n$, where $(e, n)$ is $A$ 's $R S A$ public key, and $g$ is an appropriate one-way hash function.

Part II.

When customer $P$ wants bank $A$ to issue an electronic coin worth one dollar $C$ which corresponds to $e_{A}^{\prime}, P$ conducts the following protocol with $A$ (Precisely, an electronic coin is $\left(C,\left\{X_{i}\right\}\right)$. For simplicity, however, we call $C$ an electronic coin.): (Step 1) $P$ chooses random values $R_{i}(i=1, \ldots, K / 2)$, and forms and sends $Z$ to A.

$$
\begin{gathered}
Z=F_{e_{\Lambda}^{\prime}}\left(X_{1}\|\ldots\| X_{K / 2} \| B\right), \\
X_{i}=R_{i}^{L_{i}} \bmod N_{i} \quad \text { for } 1 \leq i \leq K / 2 .
\end{gathered}
$$

(Step 2) $A$ gives $D_{e^{\prime}}(Z)$ to $P$ and charges $P$ 's account one dollar.

(Step 3) $P$ can then extract the electronic coin $C=G_{e_{A}^{\prime}}\left(X_{1}\|\ldots\| X_{K / 2} \| B\right)$.

Note: We can reduce the amount of information that $P$ posseses as follows:

In place of possessing $K / 2$ pieces of information, $\left\{X_{i} \mid 1 \leq i \leq K / 2\right\}, P$ possesses only one piece of infromation $X$. In Part II, $P$ obtains $C=G_{e_{A}^{\prime}}(X \| B)$. In Part III, we regard $X$ as $X_{i}$ for all $1 \leq i \leq K / 2$, and $P$ computes $R_{i}=X^{1 / L_{i}} \bmod N_{i}$ for $1 \leq i \leq K / 2$.

\section{Part III.}

To pay a shop $V$ one dollar, $P$ and $V$ proceed as follows:

For each $i=1,2, \ldots, K / 2$, steps $1-4$ are executed iteratedly.

(Step 1) $P$ sends $I_{i}, N_{i}, L_{i}, X_{i}$ to $V$. When $i=K / 2, P$ also sends $B$ and $C$ to $V$. (Step 2) $V$ selects a random value $E_{i} \in Z_{L_{i}}$, and sends it to $P$. When $i=K / 2, V$ verifies the validity of the signatures $B$ for $\left\{\left(I_{i}, N_{i}, L_{i}\right)\right\}$, and $C$ for $\left(X_{1}, \ldots, X_{K / 2}\right.$, $B$ ). If $B$ and $C$ are valid, $V$ selects a random value $E_{K / 2} \in Z_{L_{K / 2}}$, and sends it to $P$. Otherwise $V$ halts this protocol.

(Step 3) $P$ computes $Y_{i}=R_{i} \cdot S_{i}^{E_{i}} \bmod N_{i}$ and sends it to $V$.

(Step 4) $V$ verifies that $Y_{i}^{L_{i}} \equiv X_{i} \cdot I_{i}^{E_{i}}\left(\bmod N_{i}\right)$.

If $P$ passes this protocol successfully for all $i=1,2, \ldots, K / 2$, then $V$ accepts the electronic coin $C$ as one dollar.

Notes:

(1) This protocol can be modified in a manner similar to the parallel version of the extended Fiat-Shamir scheme.

(2) To prevent bank $A$ from crediting an invalid shop's account in Part IV, in steps 2 and 3 , we can enhance the protocol as follows:

In step $2, V$ selects a random value $d_{i}$, and sends $V$ 's identity $I D_{V}$, time $T$, and $d_{i}$ to $P$ in place of sending $E_{i} . V$ computes $E_{i}=f\left(I D_{V}\|T\| d_{i}\right)$, where $f$ is a one-way function whose output is uniformly random. In step $3, P$ also computes $E_{i}$. 
Part IV.

For bank $A$ to credit $V$ 's account by one dollar, $V$ sends the history of Part III of this protocol, $H$, to $A$, which credits $V$ 's account after verifying whether $H$ is a correct history of Part III and whether $H$ has not been stored already in $A$ 's database. If $H$ is valid, bank $A$ must store $H$ in its database.

(End of Protocol 1)

In the rest of this paper, we adopt the following notations:

(1) $\bar{C}$ represents valid cash which is correctly issued by valid bank $A$ through its designated protocol. $\bar{P}$ represents a valid customer with $\bar{C}$.

(2) $\widetilde{C}$ represents invalid cash generated through an arbitrary polynomial-time algorithm of an invalid customer $\tilde{P}$.

(3) $C$ represents either $\bar{C}$ or $\widetilde{C}$. $P$ represents either $\bar{P}$ or $\widetilde{P}$.

(4) $\bar{H}$ represents the valid history of the protocol between a valid customer $\bar{P}$ and a valid shop $\bar{V}$.

(5) $\widetilde{H}$ represents an invalid history generated through an arbitrary polynomialtime algorithm of an invalid shop $\tilde{V}$.

(6) $H$ represents either $\bar{H}$ or $\widetilde{H} . V$ represents either $\bar{V}$ or $\tilde{V}$.

Definition 5. The untraceable electronic cash system (Protocol 1 ) is secure if the following conditions are satisfied:

- Completeness: Any valid cash $\bar{C}$ is accepted as valid by any shop $\bar{V}$ through part III of Protocol 1. Any valid history $\bar{H}$ is accepted as valid by bank $\bar{A}$.

- Soundness: Any invalid cash $\widetilde{C}$ is accepted by any shop $\bar{V}$ through part III of Protocol 1 with negligible probability. Any invalid history $\widetilde{H}$ is accepted by bank $\bar{A}$ with negligible probability.

- Untraceability: For any $V, I_{i}, N_{i}, L_{i}$, and $t$, there exists a polynomial-time probabilistic Turing machine $M_{V}$ such that $\left(C,\left\{X_{i}\right\}, B\right),\left(\bar{P}\left(\left\{S_{i}\right\}\right), V(t)\right)\left(\left\{I_{i}\right.\right.$, $\left.\left.\left.N_{i}, L_{i}\right\}\right)\right)$ and $M_{V}^{A}\left(\left\{I_{i}, N_{i}, L_{i}\right\}, t\right)$ are polynomially indistinguishable.

- Unreusability: There exists a polynomial-time probabilistic Turing machine $M_{\bar{V}_{1}, \bar{V}_{2}}$ (with complete control over $\bar{V}_{1}$ and $\bar{V}_{2}$ ) such that if $\bar{P}$ 's coin $\bar{C}$ is used twice through part III of Protocol 1 to $\bar{V}_{1}$ and $\bar{V}_{2}$ respectively, then $M_{\bar{V}_{1}}, \bar{V}_{2}$, on input these histories $\bar{H}_{1}$ and $\bar{H}_{2}$, outputs at least one piece of information $S_{i}=I D_{P}\left\|a_{i}\right\| G_{e_{P}}\left(I D_{P} \| a_{i}\right)(i \in\{1, \ldots, K / s\})$ with overwhelming probability.

Note: Informally, untraceability means that when a valid coin $\bar{C}$ is used only once, the identity of the customer who uses $\bar{C}$ cannot be revealed by anyone. Unreusability means that when a valid coin $\bar{C}$ is used twice, bank $A$ can obtain the identity of the customer who uses $\bar{C}$ with overwhelming probability.

Theorem 2. Protocol 1 is secure if the following two assumptions are satisfied: (Digital signature assumption) There exist a secure digital signature scheme [GoMi Ri] and a secure multiple blind digital signature scheme.

(RSA assumption) The RSA scheme is secure. And, to break the RSA scheme in which the plaintext's redundancy is 0 is as hard to break as the RSA scheme in 
which the plaintext's redundancy is less than $1 / 2$, where $S$ 's redundancy is $r$ if $S$ is randomly selected from a source with the entropy of $(1-r)|S|$ bits. (Here, "as hard as" is defined from the viewpoint of usual "polynomial-time reduction.")

\section{Proof Sketch:}

\section{Completeness: $\bar{C}$ and $\bar{H}$ are accepted with probability 1 .}

Soundness: First, we prove the following (the soundness can be directly reduced from the folowing result if the digital signature assumption and RSA assumption are satisfied, because a polynomial-time algorithm can be constructed to break these assumptions using $M_{p}$ if $\widetilde{C}$ is accepted):

There exists a polynomial-time probabilistic Turing machine $M_{P}$ (with complete control over $P$ ) such that if $C$ is accepted to be valid by $\bar{V}$ through part III of Protocol 1 with non-negligible probability, then $M_{P}$ breaks cryptographic assumption on $A$ 's digital signatures with the public key $e_{A}^{\prime}$, and, for any positive constant $b<1, M_{P}$, on input $\left\{I_{i}\right\}$ and $b$, outputs a subset $\mathcal{S} \subset\left\{S_{i}\right\}$ such that $\# \mathcal{S} /(K / 2)>b$ with overwhelming probability, where denotes the cardinality of a subset.

If $C$ is accepted by $\bar{V}$ with non-negligible probability, then $P$ can generate $A$ 's digital signature for non-negligible fraction of the message space. Therefore, from the definition of secure blind digital signature [GoMiRi], there exists a polynomial-time probabilistic Turing machine $M_{P}$ such that $M_{P}$ breaks cryptographic assumptions of $A$ 's blind digital signature with overwhelming probability. Next we show that when $C$ is accepted by $\bar{V}$ with non-negligible probability, then, for any positive constant $b<1, M_{P}$ can output a subset $\mathcal{S} \subset\left\{S_{i}\right\}$ such that $\# \mathcal{S} /(K / 2)>b$ with overwhelming probability. Let $T$ be the truncated execution tree of $(P, \bar{V}) . T$ has $(K / 2)$ levels, and each vertex in $T$ has at most $L_{i}(=O(1))$ sons, because $\bar{V}$ may ask $L_{i}$ possible questions at each stage. A vertex is called heavy if it has at least two sons. If we can find a heavy vertex of the $i$-th level, we can compute $S_{i}$, since $L_{i}$ is prime. Then, for a positive constant $b<1$, we assume that at least $(1-b)(K / 2)$ levels have at least one non-heavy vertex. Then, the total number of leaves in $T$ is at most a negligible $\left(O\left(2^{-K}\right)\right)$ fraction of the possible leaves. Therefore, for any positive constant $b<1$, more than $b(K / 2)$ levels have all heavy vertices. Hence, we can find at least one heavy vertex in each level with all heavy vertices in polynomial-time by blind exploration of $T$, since a non-negligible fraction of the leaves is assumed to survive the truncation.

Finally, we can conclude the proof of soundness by showing in a similar manner that there exists a polynomial-time probabilistic Turing machine $M_{V}$ (with complete control $V$ ) such that if history $H$ is accepted to be valid by $\bar{A}$ through part IV of Protocol 1 with non-negligible probability, then $M_{V}$ breaks cryptographic assumption on $A$ 's digital signatures with the public key $e_{A}^{\prime}$.

Untraceability: In a manner similar to the proof of the zero-knowledgeness of the extended Fiat-Shamir scheme [OhO], it can be proven that there exists $M_{V}$ such that $\left.\left(C,\left\{X_{i}\right\}, B\right),\left(\bar{P}\left(\left\{S_{i}\right\}\right), V(t)\right)\left(\left\{I_{i}, N_{i}, L_{i}\right\}\right)\right)$ and $M_{V}^{A}\left(\left\{I_{i}, N_{i}, L_{i}\right\}, t\right)$ are polynomially indistinguishable.

Disposability: We show that when $\bar{C}$ is used twice, any $S_{i}$ cannot be revealed 
with negligible probability. Let $\mathcal{E}=\left\{E_{i} \mid E_{i}\right.$ selected by $V_{1}$ and $E_{i}$ selected by $V_{2}$ are different in part III, $1 \leq i \leq K / 2\}$. Then, for any positive constant $b<1$, $\# \mathcal{E}>b(K / 2)$ with overwhelming probability. Since $L_{i}$ is prime, we can calculate $I_{i}^{1 / L_{i}} \bmod N_{i}$ for $i$ whose $E_{i}$ is in $\mathcal{E}$. Therefore, to make all $S_{i}$ 's be concealed from anyone, at least $b(K / 2)$ blind candidates $W_{i}$ must be invalid in part I, and all these invalid candidates must not be selected in $U$. Hence, all $S_{i}$ 's can be concealed from anyone with probability $2^{-b(K / 2)}$ (or negligible probability). In other words, if $\bar{C}$ is used $t$ wice, at least one piece of information $S_{i}$ can be revealed with overwhelming probability. QED

\section{Transferable Untraceable Electronic Cash}

In this section, we propose an electronic cash scheme satisfying the criterion of tranferability in addition to untraceability and unreusability.

Protocol 2. (Transferable untraceable electronic cash)

This protocol is constructed based on Protocol 1. Therefore, undefined notations and procedures follow the definitions in Protocol 1. To simplify the description of this protocol, we suppose a case where bank $A$ issues one dollar electronic coin $C$ to customer $P_{1}$, who transfers $C$ to customer $P_{2}$, and $P_{2}$ uses $C$ at shop $V$.

Part I.

When customers $P_{1}$ and $P_{2}$ open their accounts at bank $A, A$ issues electronic licenses $B^{(j)}$ to a customer $P_{j}(j=1,2)$. Hereafter, in this protocol, $x^{(j)}$ means $x$ of $P_{j}$, where variable $x$ follows the definition in Protocol 1.

\section{Part II.}

Suppose that customer $P_{1}$ have bank $A$ issue an electronic coin worth one dollar C.

\section{Part III.}

To transfer $C$ to another customer $P_{2}, P_{1}$ and $P_{2}$ proceeds as follows:

(Step 1) $P_{1}$ and $P_{2}$ follow the same protocol as that for $P_{1}$ to pay shop $P_{2}$ one dollar (Part III of Protocol 1).

(Step 2) $P_{1}$ sends a certification $T$ that denotes the transfer of $C$ from $P_{1}$ to $P_{2}$. For example, $P_{1}$ sends a digital signature $G_{\left(N_{1}^{(1)}, L_{1}^{(1)}\right)}\left(C \| B^{(2)}\right)($ e.g., $T=g(C \|$ $\left.\left.B^{(2)}\right)^{1 / L_{1}^{(1)}} \bmod N_{1}^{(1)}\right)$.

$P_{2}$ generates

$$
R_{i}^{(2)}=\left(X_{i}^{(1)}\right)^{1 / L_{i}^{(2)}} \bmod N_{i}^{(2)} \quad \text { for } 1 \leq i \leq K / 2 .
$$

If $P_{2}$ accepts $P_{1}$ 's electronic coin $C$ through step 1 and verifies the validity of $T$, then $P_{2}$ pays one dollar to $P_{1}$.

Part IV.

To pay shop $V$ one dollar, $P_{2}$ and $V$ proceeds as follows:

(Step 1) $P_{2}$ sends the history of Part III of this protocol, $H^{(1)}$, to $V$. $V$ checks the validity of $H^{(1)}$. 
(Step 2) $P_{2}$ follows Part III of Protocol 1 with shop $V$ to pay $C$.

\section{Part $V$.}

To have bank $A$ credit $V$ 's account by one dollar, $V$ sends the history of Part IV of this protocol, $B^{(2)}$, to $A$, which credits $V$ 's account after verifying whether $H^{(2)}$ is a correct history of Part IV and whether $H^{(2)}$ has not been stored already in $A$ 's database. If $H^{(2)}$ is valid, bank $A$ must store $H^{(2)}$ in its database.

(End of Protocol 2)

Note: Informally, Protocol 2 is secure if the following conditions are satisfied. The formal definition and proof regarding the security of Protocol 2 will be shown in the final paper.

- Completeness: Any (original/transfered) valid cash $\bar{C}$ of $\bar{P}_{1}$ and $\bar{P}_{2}$ is accepted to be valid by $\bar{P}_{2}$ and any shop $V$, respectively. Any valid history $\bar{H}^{(2)}$ is accepted to be valid by bank $\bar{A}$.

- Soundness: Any (transfered) invalid cash $\tilde{C}$ is accepted by $P_{2}$ and any shop $\bar{V}$ with negligible probability. Any invalid history $\widetilde{H}^{(2)}$ is accepted by any bank with negligible probability.

- Untraceability: When a valid coin $\bar{C}$ is used only once, any knowledge about the identity of the customer who uses $\bar{C}$ cannot be revealed by anyone.

- Unreusability: When coin $\bar{C}$ is used twice correctly by a customer, bank $\bar{A}$ can obtain the identity of the customer with overwhelming probability. When coin $\bar{C}$ is used twice correctly by two different customers $\bar{P}_{1}$ and $\bar{P}_{2}$, bank $\bar{A}$ can obtain the identity of $\bar{P}_{1}$ with overwhelming probability.

\section{Untraceable Electronic Coupon Tickets}

In this section, we also propose another type of untraceable electronic cash (untraceable electronic coupon ticket) with the following property in addition to those of the untraceable electronic cash: The value of one piece of electronic cash can be subdivided into several pieces. For example, a user with a piece of electronic cash worth $\$ 100$ could subdivide it into 100 pieces of cash worth $\$ 1$. Here, the data size of 100 coupon tickets is comparable to one piece of electronic cash.

If we add the notion of transferability (criterion (3)), transferable untraceable electronic coupon ticket could be constructed in a way similar to the transferable untraceable electronic cash.

Protocol 3. (Untraceable electronic coupon ticket) This protocol is constructed based on Protocol 1. Therefore, undefined notations and procedures follow the definitions in Protocol 1.

\section{Part I.}

To obtain license $B$ from bank $A$, customer $P$ follows the same protocol with $A$ as Part I of Protocol 1.

Part II.

To obtain a piece of information (electronic coupon tickets), $C$, which is 100 tickets 
each worth $\$ 1$, customer $P$ conducts the same protocol with bank $A$ as Part II of Protocol 1. Here, the value of $e^{\prime}$ indicates the value and type of electronic coupon tickets $C$ (e.g., 100 tickets each worth $\$ 1$ ).

Part III.

To pay shop $V$ the $j$-th one dollar ticket $(1 \leq j \leq 100), P$ and $V$ proceeds as follows:

First, $P$ generates $X_{i}^{\langle j\rangle}=f_{j}\left(X_{i}\right)$ and $R_{i}^{\langle j\rangle}=\left(X_{i}^{\langle j\rangle}\right)^{1 / L_{i}} \bmod N_{i}$. Here, $f_{j}(x)$ means a one-way function with a parameter $j$. For example, we can construct $f_{j}$ by a one-way function $f$ such that

$$
f_{j}(x)=f(x \| j), \quad \text { or } \quad f_{j}(x)=f\left(x \| 1^{j}\right) .
$$

For each $i=1,2, \ldots, K / 2$, steps $1-4$ are executed iteratedly.

(Step 1) and (Step 2) are the same as those of Protocol 1.

(Step 3) and (Step 4) are the same as those of Protocol 1 except replacing $X_{i}$ and $R_{i}$ by $X_{i}^{\langle j\rangle}$ and $R_{i}^{\langle j\rangle}$, respectively. Here, $P$ also sends $j$, and $V$ checks that $1 \leq j \leq 100$ and generates $X_{i}^{<j>}=f_{j}\left(X_{i}\right)$.

If $P$ passes this protocol successfully for all $i=1,2, \ldots, K / 2$, then $V$ accepts the $j$-th one dollar ticket of the $\$ 100$ electronic coupon tickets $C$.

Part IV.

For bank $A$ to credit $V$ 's account by one dollar, $V$ sends the history of Part III of this protocol, $H^{\langle j\rangle}$, to $A$, which credits $V$ 's account after verifying whether $H^{\langle j\rangle}$ is a correct history of Part III and whether $B^{\langle j\rangle}$ has not been stored already in $A$ 's database. If $H^{\langle j\rangle}$ is valid, bank $A$ must store $H^{\langle j\rangle}$ in its database.

(End of Protocol 3)

\section{Conclusion}

In this paper, we have proposed a new type of authenticatication, disposable zero-knowledge authentication, and described its applications to untraceable electronic cash schemes. To find other applications of the disposable zero-knowledge authentication remains further work. We improved the efficiency of our scheme by reducing the degree of untraceability. We will concentrate on improving the efficiency of these schemes with perfect untraceability in further work. In the proof of the security of these protocols, we have supposed some assumptions. To reduce these assumptions to even more fundamental assumptions remains an open challenge.

Acknowledgements: We would like to thank Eugène van Heyst for many valuable comments and suggestions on the earlier version. We would also like to thank anonymous referees for their helpful comments. 


\section{References}

[B] J.C.Benaloh, "Cryptographic capsules: A disjunctive promitive for interactive protocols," The Proc. of Crypto'86, pp.213-222 (1986)

[BC] G.Brassard and C.Crépeau, "Non-Transitive Transfer of Confidence: A perfect Zero-Knowledge Interactive Protocol for SAT and Beyond," The Proc. of FOCS'86, pp.188-195 (1986)

[BCC] G.Brassard, D.Chaum, and C.Crépeau, "Minimum Disclosure Proofs of Knowledge," Journal of Computer and System Sciences, Vol.37, pp.156189 (1988)

[Ch] D.Chaum, "Security without Identification: Transaction Systems to Make Big Brother Obsolete," Comm. of the ACM, 28, 10, pp.10301044 (1985)

[Cr] C.Crépeau, "A zero-knowledge poker protocol that achieves confidentiality of the players' strategy or How to achieve an electronic poker face," The Proc. of Crypto'86, pp.239-247 (1986)

[CFN] D.Chaum, A.Fiat and M. Naor, "Untraceable Electronic Cash," to appear in the Proc. of Crypto'88 (1988)

[D] I.B.Damgård, "Payment Systems and Credential Mechanisms with Provable Security Against Abuse by Individuals," to appear in the Proc. of Crypto'88 (1988)

[FFS] U.Feige, A.Fiat and A.Shamir, "Zero Knowledge Proofs of Identity," The Proc. of STOC, pp.210-217 (1987)

[FS] A.Fiat and A.Shamir, "How to Prove Yourself," The Proc. of Crypto'86, pp.186-199 (1986)

[GM] S.Goldwasser, and S.Micali, "Probabilistic Encryption," Journal of Computer and System Science, Vol.28, No.2 (1984)

[GMR] S.Goldwasser, S.Micali, and C.Rackoff, "Knowledge Complexity of Interactive Proofs," The Proc. of STOC, pp291-304 (1985)

[GoMiRi] S.Goldwasser, S.Micali, and R.Rivest, "A Digital Signature Scheme Secure Against Adaptive Chosen-Message Attacks," SIAM J.Compt., 17, 2, pp.281-308 (1988)

[GMW] O.Goldreich, S.Micali, and A.Wigderson, "Proofs that Yield Nothing But their Validity and a Methodology of Cryptographic Protocol Design," The Proc. of FOCS, pp.174-187 (1986)

[GQ] L.C.Guillou, and J.J.Quisquater, "A Practical Zero-Knowledge Protocol Fitted to Security Microprocessors Minimizing Both Transmission and Memory," The Proc. of Eurocrypto'88, pp.123-128 (1988)

[MS] S.Micali, and A.Shamir, "An Improvement of The Fiat-Shamir Identification and Signature Scheme," The Proc. of Crypto'88 (1988)

[OhO] K.Ohta, and T.Okamoto "A Modification of the Fiat-Shamir Scheme," to appear in the Proc. of Crypto'88 (1988)

[OkO] T.Okamoto, and K.Ohta "Divertible Zero-Knowledge Interactive Proofs and Commutative Random Self-Reducible," to appear in the Proc. of Eurocrypt'89 (1989) 
[TW] M.Tompa and H.Woll, "Random Self-Reducibility and Zero Knowledge Interactive Proofs of Possession of Information," The Proc. of FOCS, pp472-482 (1987)

[Y] A.C. Yao: Theory and Applications of Trapdoor Functions, The Proc. of FOCS, pp.80-91 (1982) 